\title{
APLICAÇÃO CONJUNTA DE HERBICIDAS E INSETICIDAS NA CULTURA DO MILHO $\left({ }^{(1)}\right.$
}

\author{
MARCELO NICOLAI $\left({ }^{2}\right)$; SAUL JORGE PINTO DE CARVALHO $\left(3^{*}\right)$; \\ RAMIRO FERNANDO LÓPEZ-OVEJERO $\left({ }^{4}\right)$; PEDRO JACOB CHRISTOFFOLETI $\left({ }^{5}\right)$
}

\begin{abstract}
RESUMO
Este trabalho foi desenvolvido com o objetivo de verificar a seletividade de herbicidas aplicados em pós-emergência da cultura do milho (Zea mays L.) quando misturados em tanque com inseticidas utilizados no controle de Spodoptera frugiperda (J.E. Smith). O experimento foi realizado com milho safrinha, semeado em fevereiro de 2004, usando o híbrido AGN 2012, em sistema convencional. O delineamento experimental foi do tipo blocos ao acaso com parcelas subdividas no modelo de testemunha dupla, quatro repetições e 15 tratamentos. Os tratamentos foram constituídos pela combinação de três níveis do fator herbicida $\left(\mathrm{g} \mathrm{ha}^{-1}\right)$ : mesotrione $(120)+$ atrazine $(1500)+$ Assist $0,5 \%$, nicosulfuron $(20)+$ atrazine $(1500)$ e testemunha capinada; com cinco níveis do fator inseticida: lambdacyhalothrin (10), chlorpyrifos CE (240), chlorpyrifos EC (225), lambdacyhalothrin + thiamethoxam $(12,5+5,5)$ e testemunha sem aplicação de inseticidas. Foram observados sintomas de fitotoxicidade moderados para os tratamentos com mesotrione e severos para os de nicosulfuron causados pela interação desses herbicidas com o inseticida chlorpyrifos (formulações CE e EC) que, contudo, não se refletiram em reduções de produtividade.
\end{abstract}

Palavras-chave: interação, fitotoxicidade, rendimento, Zea mays.

\section{ABSTRACT \\ JOINT APLICCATION OF HERBICIDES AND INSECTICIDES IN THE CORN CROP}

The selectivity of post-emergence applied herbicides in the corn crop (Zea mays L.) when mixed in tank with insecticides used to control Spodoptera frugiperda (J.E. Smith) was verified. The experiment was carried out in off-season maize, seeded in February 2004, using the hybrid AGN 2012, in conventional system. The experimental design was randomized blocks with split-plots scheme, on double check model, four replicates and 15 treatments. The treatments were constituted by the combination of three levels of the factor herbicide $\left(\mathrm{g} \mathrm{ha}^{-1}\right)$ : mesotrione $(120)+$ atrazine $(1500)+$ Assist $0,5 \%$, nicosulfuron $(20)+$ atrazine (1500) and hand-weeded checks; with five levels of the factor insecticide: lambdacyhalothrin (10), chlorpyrifos CE (240), chlorpyrifos EC (225), lambdacyhalothrin + thiamethoxam $(12,5+5,5)$ and checks without insecticide application. Moderate phytotoxic symptoms were observed to mesotrione treatments and severe symptoms were observed to nicosulfuron caused by the interaction of these herbicides with the insecticide chlorpyrifos (formulations CE and EC). No yield losses were recorded as due to phytotoxicity.

Key words: interaction, phytotoxicity, yield, Zea mays.

( $\left.{ }^{1}\right)$ Parte da dissertação de mestrado do primeiro autor. Recebido para publicação em 29 de novembro de 2004 e aceito em 23 de junho de 2006.

$\left(^{2}\right)$ Doutorando da ESALQ/USP. Bolsista do CNPq. E-mail: marcelon@esalq.usp.br.

$\left.{ }^{3}\right)$ Mestrando da ESALQ/USP. Bolsista FAPESP. E-mail: sjpcarvalho@yahoo.com.br. *Autor correspondente.

$\left({ }^{4}\right)$ Doutorando da ESALQ/USP. E-mail: rfloveje@esalq.usp.br

$\left({ }^{5}\right)$ Departamento de Produção Vegetal, ESALQ/USP. Caixa Postal 9, 13418-900 Piracicaba (SP). E-mail: pjchrist@esalq.usp.br. 


\section{INTRODUÇÃO}

O milho (Zea mays L.) é uma das principais culturas agrícolas cultivadas no Brasil, não somente pelo aspecto quantitativo, mas também por sua importância estratégica, visto que é a base da alimentação animal e, conseqüentemente, humana. Dentre os fatores bióticos que interferem na cultura do milho, as plantas daninhas podem causar perdas de rendimento de até $70 \%$. Neste caso, o controle de plantas daninhas é uma necessidade de ordem econômica, em que o método químico, por meio de herbicidas, é o mais viável (LÓPEZ-OvEJERo et al., 2003a).

A seletividade dos herbicidas é a base para o sucesso do controle químico de plantas daninhas na produção agrícola e pode ser definida como a medida da resposta diferencial das espécies de plantas à aplicação de uma determinada molécula (Oliveira JúnIOR, 2001). Dentre os fatores de seletividade relacionados às características das plantas, direta ou indiretamente, o metabolismo diferencial de herbicidas destaca-se como um dos mais importantes (Cole, 1994). Dessa forma, a adição de adjuvantes, nutrientes ou inseticidas à calda contendo herbicida pode modificar a capacidade da planta em tolerá-lo, tendo por conseqüência a perda da seletividade ou da eficácia no controle das plantas daninhas (GASSEN, 2002).

Dentre os herbicidas recomendados para a cultura do milho, aqueles do grupo das sulfoniluréias necessitam da observação de fatores que, quando negligenciados, podem interferir em sua seletividade à cultura, como: o híbrido utilizado, momento fenológico de aplicação, intervalo entre a aplicação do herbicida e a adubação nitrogenada de cobertura ou aplicação do inseticida organo-fosforado (LóPEZOvejero et al., 2003b).

Nos agroecossistemas brasileiros onde se cultiva milho, além da constante presença das plantas daninhas, a ocorrência de Spodoptera frugiperda (J.E. Smith) é generalizada, prejudicando o desenvolvimento da planta e se caracterizando como a principal praga da cultura, atacando-a em todas as fases de seu desenvolvimento (CRUZ, 2003). A lagarta-do-cartucho, como é conhecida popularmente, pode gerar grandes perdas na produção e na qualidade dos grãos, sendo controlada por meio da aplicação de inseticidas do grupo dos piretróides e fosforados (Омото et al., 2002).

A condição de um sistema produtivo, sobretudo quando em áreas extensas, nem sempre possibilita a adoção de todas as recomendações agrícolas no momento correto. Assim, a aplicação de herbicidas e inseticidas, por vezes, pode ocorrer em intervalo muito curto de tempo ou mesmo simultaneamente. $\mathrm{Na}$ literatura, existem diversos trabalhos científicos relatando a interação negativa entre herbicidas pertencentes ao grupo dos inibidores da enzima ALS com inseticidas organofosforados na cultura de milho (Morton et al., 1991; Morton et al., 1994; SiminsZKY et al., 1995; SiLva et al., 2005a;b).

Considerando a necessidade da utilização simultânea de mais de um defensivo durante o desenvolvimento da cultura, tornam-se necessários estudos sobre as possíveis interações entre os produtos agrícolas a fim de a orientar o produtor com relação ao uso adequado dos diferentes herbicidas que podem ser aplicados em pós-emergência da cultura do milho. Esse trabalho foi desenvolvido com o objetivo de avaliar a seletividade dos herbicidas mesotrione + atrazine e nicosulfuron + atrazine quando aplicados na condição de mistura em tanque com os inseticidas lambdacyhalothrin, chlorpyrifos CE, chlorpyrifos EC e lambdacyhalothrin + thiamethoxam, à cultura do milho.

\section{MATERIAL E MÉTODOS}

Este trabalho foi realizado em área experimental situada no município de Piracicaba, SP $\left(22^{\circ} 42^{\prime} 30^{\prime \prime} \mathrm{S}, 47^{\circ} 38^{\prime} 00^{\prime \prime} \mathrm{O}\right)$, na Fazenda 'Areão', entre fevereiro e junho de 2004. A cultura do milho foi desenvolvida em condição de "safrinha", em área sem irrigação e com preparo de solo do tipo convencional. O solo da área é classificado como Nitossolo Vermelho Eutroférrico Latossólico (EMBRAPA, 1999), de textura argilosa e com as propriedades químicas apresentadas na tabela 1.

Tabela 1. Análise química do solo (Nitossolo Vermelho Eutroférrico latossólico) da área experimental. Fazenda "Areão", Piracicaba, 2004.

\begin{tabular}{|c|c|c|c|c|c|c|c|c|c|c|c|c|}
\hline$\overline{\mathrm{pH}}$ & M.O. & $\mathrm{P}$ & $S$ & $\mathrm{~K}$ & $\mathrm{Ca}$ & $\mathrm{Mg}$ & $\mathrm{Al}$ & $\mathrm{H}+\mathrm{Al}$ & SB & $\mathrm{T}$ & $\mathrm{V}$ & $\mathrm{m}$ \\
\hline $\mathrm{CaCl}_{2}$ & $\mathrm{~g} \mathrm{dm}^{-3}$ & \multicolumn{2}{|c|}{$-\mathrm{mg} \mathrm{dm}^{-3}--$} & \multicolumn{7}{|c|}{ mmolc $\mathrm{dm}^{-3}$} & \multicolumn{2}{|c|}{$\%-$} \\
\hline 5,5 & 24 & 44 & 28 & 9,5 & 67 & 31 & 0 & 28 & 107 & 135 & 79 & 0 \\
\hline
\end{tabular}

$\mathrm{S}=\mathrm{NH}_{4} \mathrm{OAc}$ 0,5 N em HOAc 0,25N (10 ml TFSA/25 ml). Análise realizada pelo Departamento de Solos e Nutrição de Plantas da ESALQ/USP. 
A partir da análise química do solo (Tabela 1) e das recomendações da literatura visando a produtividades entre 6 e $8 \mathrm{t} \mathrm{ha}^{-1}$ (RAIJ e CANTARELla, 1996), aplicaram-se $350 \mathrm{~kg} \mathrm{ha}^{-1}$ do adubo formulado 0828-16 como adubação de base. A semeadura do híbrido AGN 2012 foi realizada em 2 de fevereiro de 2004 obtendo-se densidade final de 60.000 plantas ha ${ }^{-1}$, após desbaste. Quando as plantas estavam em estádio fenológico de oito folhas realizou-se a adubação de cobertura com sulfato de amônio $\left(62 \mathrm{~kg} \mathrm{ha}^{-1}\right.$ de N) a lanço, em área total. Os dados meteorológicos referentes ao período e local em que a cultura esteve a campo estão apresentados na figura 1.

O delineamento experimental utilizado foi do tipo blocos ao acaso, em esquema fatorial $3 \times 5$ e quatro repetições. As unidades experimentais constaram de cinco linhas da cultura, espaçadas em $0,8 \mathrm{~m}$ com $4,0 \mathrm{~m}$ de comprimento, o que totalizou 16 $\mathrm{m}^{2}$ de área total. Todas as parcelas foram mantidas sem a presença de plantas daninhas durante todo o experimento por meio de capinas manuais. Foram realizadas, também, duas aplicações de delthametrina a $5 \mathrm{~g} \mathrm{ha}^{-1}$ para o controle de lagartado-cartucho, sendo a primeira quando a cultura estava com duas folhas verdadeiras e a segunda com oito folhas verdadeiras.
Os tratamentos utilizados foram decorrentes da combinação entre três níveis do fator herbicida ( $\mathrm{g} \mathrm{ha}^{-1}$ do ingrediente ativo): mesotrione (120) + atrazine (1.500) + Assist 0,5\%, nicosulfuron (20) + atrazine (1.500) e testemunha capinada durante todo o experimento; e cinco níveis do fator inseticida ( $\mathrm{g} \mathrm{ha}^{-1}$ do ingrediente ativo): lambdacyhalothrin (10), chlorpyrifos (240, concentrado emulsionável - CE), chlorpyrifos fersol (225, emulsão concentrada - EC), lambdacyhalothrin + thiamethoxam $(12,5+5,5)$ e testemunha sem aplicação de inseticidas. Os tratamentos foram alocados no campo segundo o modelo de testemunhas duplas, de tal forma que sempre adjacente a uma parcela tratada com qualquer um dos produtos existia, pelo menos, uma parcela testemunha.

Todos os tratamentos foram aplicados em 23 de fevereiro de 2004, 15 dias após a emergência das plantas, as quais possuíam de quatro a cinco folhas verdadeiras. As aplicações foram realizadas com um pulverizador costal pressurizado por $\mathrm{CO}_{2}$ à pressão constante de $2,5 \mathrm{kgf} \mathrm{cm}^{-2}$, equipado com barra de aplicação com quatro pontas do tipo leque, modelo Teejet XL 110.02 VS, calibrado para um volume de calda proporcional a $200 \mathrm{~L} \mathrm{ha}^{-1}$.

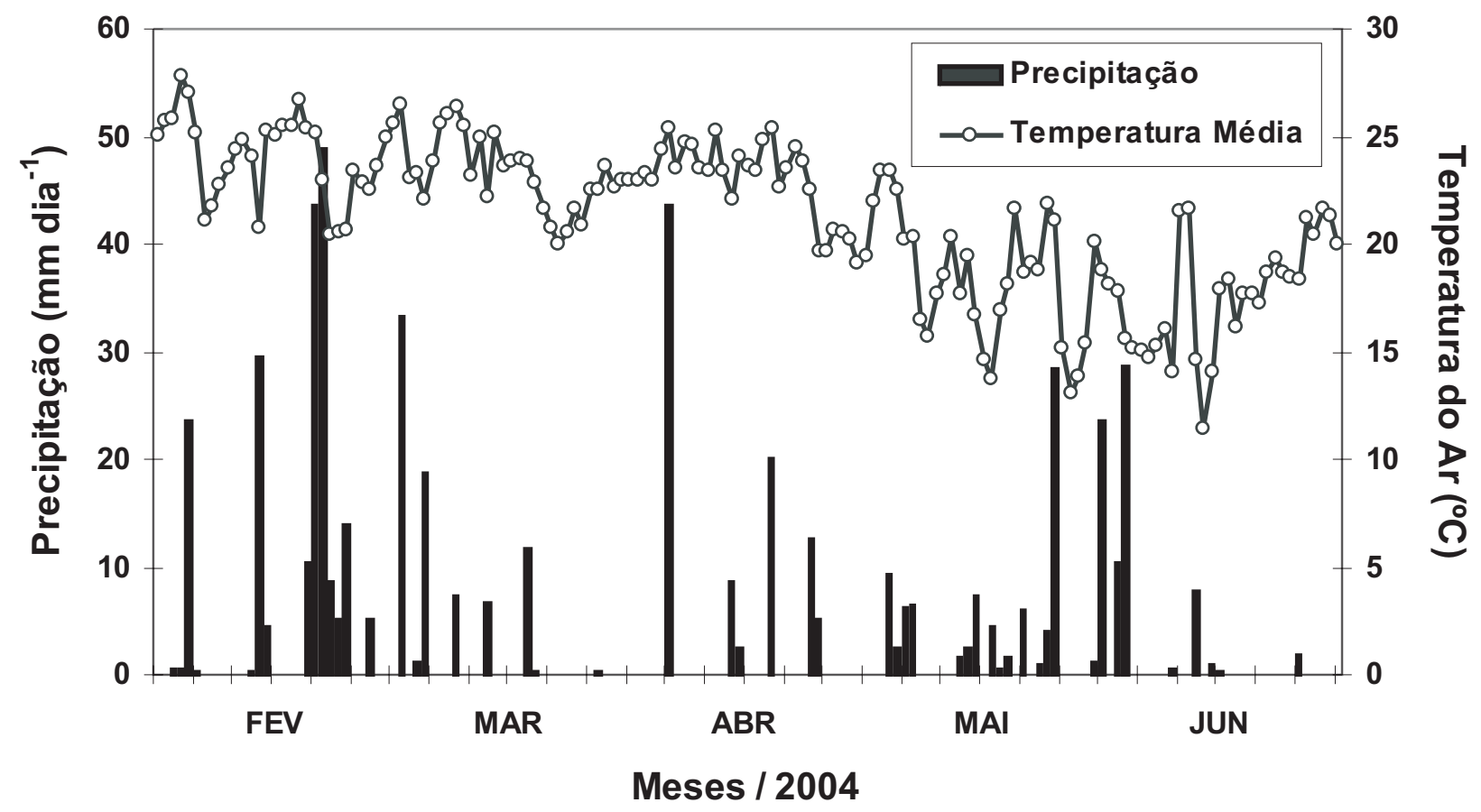

Figura 1. Precipitação pluvial $(\mathrm{mm})$ e temperatura média $\left({ }^{\circ} \mathrm{C}\right)$ diárias para o período de realização do experimento. Piracicaba, 2004. 
As variáveis avaliadas foram: fitotoxicidade aos 7, 14, 21 e 28 dias após aplicação (DAA); altura das plantas de milho por ocasião do florescimento; massa de mil grãos e rendimento $\left(\mathrm{t} \mathrm{ha} \mathrm{h}^{-1}\right)$. A fitotoxicidade foi avaliada por meio de uma escala percentual de danos causados à morfologia das plantas de milho, variando entre 0 (ausência de danos) e $100 \%$ (morte das plantas). A altura das plantas foi mensurada aos 70 dias após emergência (florescimento), medindo-se dez plantas por parcela, do nível do solo à base da panícula em floração.

A colheita da cultura foi realizada em 23 de junho de 2004. O rendimento foi obtido com a retirada das espigas da segunda e terceira linhas da parcela, em seus 3,0 m centrais, totalizando-se uma área útil de $4,8 \mathrm{~m}^{2}$. As espigas foram despalhadas e debulhadas mecanicamente, separarando-se os grãos de cada parcela, os quais foram pesados. Nesse momento, cada amostra teve sua umidade (\%) mensurada e corrigida para $14 \%$ de umidade. De cada parcela, uma amostra de mil grãos foi retirada, pesada e também teve sua umidade corrigida para $14 \%$.

O modelo de experimento com testemunhas duplas foi analisado estatisticamente segundo o delineamento de parcelas subdivididas, em que no âmbito de parcelas consideraram-se os níveis dos fatores herbicida e inseticida, bem como sua combinação, e no âmbito de subparcelas considerouse a existência do tratamento ou de sua testemunha respectiva. Sempre que um tratamento possuía duas parcelas testemunhas adjacentes, adotou-se a média das duas como valor de comparação (FAGLIARI et al., 2001; Constantin et al., 2003).

A variável fitotoxicidade foi analisada segundo modelo fatorial, desconsiderando-se o esquema de parcelas subdivididades, uma vez que, em um experimento de seletividade, a fitotoxicidade das testemunhas é sempre zero. Todas as análises foram feitas por meio da aplicação do teste $\mathrm{F}$ sobre a análise da variância, seguido de teste Tukey com 5\% de significância para a comparação das médias.

\section{RESULTADOS E DISCUSSÃO}

Em todas as avaliações de fitotoxicidade foram identificadas interações entre os níveis de tratamentos dos fatores herbicida e inseticida. Aos 7 DAA, verificaram-se diferenças (fitotoxicidade severa) entre o inseticida chlorpyrifos aplicado em mistura com nicosulfuron + atrazine e os demais tratamentos, independente da formulação desse organofosforado, provocada pela interação sinergística entre essas moléculas. Os tratamentos que envolveram o herbicida mesotrione também provocaram fitotoxicidade às plantas, contudo em menor grau que os tratamentos que envolveram o nicosulfuron (Tabela 2).

Estes resultados são semelhantes aos encontrados por MoRTON et al. (1991), que observaram efeitos severos de fitotoxicidade de nicosulfuron em milho, quando o organo-fosforado terbufós foi aplicado no sulco de plantio. Também, KwON e PenNeR (1995) observaram que misturas de herbicidas do grupo das sulfoniluréias com terbufós resultaram em interação sinérgica fitotóxica altamente significativa. Todavia, quando foram utilizados os inseticidas chlorpyrifos, tefluthrin e carbofuran os efeitos de fitotoxicidade do nicosulfuron sobre o milho foram menos significativos (MORTON et al., 1994).

Aos 14 e 21 DAA, os tratamentos inseticidas com lambdacyhalothrin (isolado ou em mistura com thiamethoxan) foram os que provocaram menor fitotoxicidade às plantas, mesmo quando em mistura com mesotrione + atrazine ou nicosulfuron + atrazine. Nestas datas, de modo geral, a fitotoxicidade observada para a interação dos tratamentos inseticidas com os tratamentos herbicidas foi menor, quando comparada com a avaliação de 7 DAA (Tabela 2). Aos 28 DAA, verificou-se a completa recuperação da cultura para todos os tratamentos, sendo os sintomas visuais de fitotoxicidade quase imperceptíveis, exceto para as misturas de chlorpyrifos CE e chlorpyrifos EC com os herbicidas nicosulfuron + atrazine que ainda apresentavam 11,5 e 13,5\% de fitotoxicidade respectivamente (Tabela 2).

Os sintomas de fitotoxicidade causados pela mistura de herbicidas e inseticidas ao híbrido de milho AGN 2012 se caracterizaram por uma clorose inicial (amarelecimento ou branqueamento) próximas do cartucho das folhas e redução da altura de plantas, sendo esses sintomas mais evidentes nas plantas pulverizadas com misturas de nicosulfuron e mesotrione com o inseticida chlorpyrifos, nas duas formulações. Os sintomas de clorose presentes são semelhantes aos descritos por SiminszKY et al. (1995), resultantes da interação de nicosulfuron e terbufós.

Quanto à tolerância do híbrido de milho à mistura em tanque dos herbicidas e inseticidas, observou-se recuperação gradativa das plantas em função do tempo decorrido após a avaliação de 7 DAA, exceto para os tratamentos com chlorpiryfos, cuja fitotoxicidade permaneceu até a avaliação de 28 DAA (Tabela 2). Essa recuperação pode ser atribuída às características genéticas do híbrido, associada a adequadas condições meteorológicas para o bom desenvolvimento da cultura e conseqüente metabolização dos produtos aplicados. Segundo 
SIMINSKI et al. (1995), há diferença de sensibilidade de variedades de milho ao nicosulfuron e resulta, em parte, do metabolismo diferencial da molécula e, principalmente, da sensibilidade diferencial da acetolactato sintase (ALS), que é a enzima-alvo.

Apesar dos severos sintomas de intoxicação causados às plantas de milho pelas misturas de herbicidas com inseticidas organofosforados, observou-se que a altura de plantas no florescimento $(\mathrm{m})$, rendimento $\left(\mathrm{t} \mathrm{ha}^{-1}\right)$ e massa de mil grãos (g) não foram afetados (Tabela 3).

Estes resultados demonstram que, apesar da redução da seletividade à cultura do milho causada pela adição de inseticidas à calda de aplicação de herbicidas, verificou-se recuperação da cultura, pois os componentes de produção não foram afetados. Esse fato evidencia a necessidade de recuperação do híbrido de milho utilizado em relação aos efeitos das misturas, que pode ser dependente da fertilidade do solo (Tabela 1), condições meteorológicas do ano agrícola (Figura 1) e demais práticas e fatores de produção que favoreçam a cultura.
Estes resultados estão em discordância daqueles encontrados por Biediger et al. (1992), que observaram em combinações de inseticidas organofosforados como disulfuton, fonofós, isazofós ou terbufós com primisulfuron (sulfoniluréia) interações sinergísticas, provocando injúrias foliares (clorose) e radiculares, redução de altura e perdas na produção final. Também, Silva et al. (2005a) verificaram que a interação fitotóxica de nicosulfuron com chlorpyrifos em milho híbrido P30F80 acarretou redução no número de espigas e no rendimento de grãos. Ainda, a aplicação de chlorpyrifos, em mistura com nicosulfuron não comprometeu o controle químico das plantas daninhas; contudo, a intoxicação causada pela mistura desfavoreceu o controle cultural. SiLva et al. (2005b), por sua vez, observaram a necessidade do intervalo mínimo de cinco dias entre a aplicação do herbicida e do inseticida para redução da interação fitotóxica sobre as plantas de milho.

Tabela 2. Médias das porcentagens de danos (fitotoxicidade) causados em milho híbrido (AGN 2012) pela aplicação de misturas de inseticidas com herbicidas aos 7, 14, 21 e 28 Dias Após Aplicação (DAA). Piracicaba, 2004

\begin{tabular}{|c|c|c|c|}
\hline \multirow{2}{*}{ Inseticidas } & \multicolumn{3}{|c|}{ Herbicidas } \\
\hline & Sem herbicida & Mesotrione + atrazine & Nicosulfuron + atrazine \\
\hline & \multicolumn{3}{|c|}{ Fitotoxicidade aos 7 DAA } \\
\hline Sem inseticida & $0,00 \mathrm{~A} \mathrm{a}$ & $3,75 \mathrm{~A} \mathrm{~b}$ & $7,00 \mathrm{~A} \mathrm{~b}$ \\
\hline Chlorpyrifos (CE) & $2,00 \mathrm{~A} \mathrm{a}$ & $10,50 \mathrm{~B} \mathrm{~b}$ & $45,00 \mathrm{C} \mathrm{c}$ \\
\hline Lambdacyhalothrin & $2,75 \mathrm{~A} \mathrm{a}$ & $4,25 \mathrm{~A} \mathrm{a}$ & $6,25 \mathrm{~A} \mathrm{a}$ \\
\hline Thiamethoxan + lambdacyhalothrin & $3,00 \mathrm{~A} \mathrm{a}$ & $3,75 \mathrm{~A} \mathrm{a}$ & $7,75 \mathrm{~A} \mathrm{~b}$ \\
\hline \multirow[t]{2}{*}{ Chlorpyrifos (EC) } & $3,00 \mathrm{~A} \mathrm{a}$ & $10,00 \mathrm{~B} \mathrm{~b}$ & $37,50 \mathrm{~B} \mathrm{c}$ \\
\hline & \multicolumn{3}{|c|}{ Fitotoxicidade aos 14 DAA } \\
\hline Sem inseticida & $0,00 \mathrm{~A} \mathrm{a}$ & $1,25 \mathrm{~A} \mathrm{a}$ & $3,75 \mathrm{~A} \mathrm{a}$ \\
\hline Chlorpyrifos (CE) & $4,50 \mathrm{~A} \mathrm{a}$ & $7,00 \mathrm{~B}$ a & $27,50 \mathrm{~B} \mathrm{~b}$ \\
\hline Lambdacyhalothrin & $2,50 \mathrm{~A} \mathrm{a}$ & $1,75 \mathrm{AB}$ a & $3,50 \mathrm{~A} \mathrm{a}$ \\
\hline Thiamethoxan + lambdacyhalothrin & $2,50 \mathrm{~A} \mathrm{ab}$ & $1,25 \mathrm{~A} \mathrm{a}$ & $6,50 \mathrm{~A} \mathrm{~b}$ \\
\hline \multirow[t]{2}{*}{ Chlorpyrifos (EC) } & $0,50 \mathrm{~A}$ a & $7,00 \mathrm{~B} \mathrm{~b}$ & $22,00 \mathrm{~B} \mathrm{C}$ \\
\hline & \multicolumn{3}{|c|}{ Fitotoxicidade aos 21 DAA } \\
\hline Sem inseticida & $0,00 \mathrm{~A} \mathrm{a}$ & $0,75 \mathrm{~A} \mathrm{a}$ & $2,00 \mathrm{~A} \mathrm{a}$ \\
\hline Chlorpyrifos (CE) & $2,75 \mathrm{~A}$ a & $5,25 \mathrm{~A}$ a & $20,25 \mathrm{~B} \mathrm{~b}$ \\
\hline Lambdacyhalothrin & $1,50 \mathrm{~A} \mathrm{a}$ & $0,75 \mathrm{~A} \mathrm{a}$ & $0,00 \mathrm{~A} \mathrm{a}$ \\
\hline Thiamethoxan + lambdacyhalothrin & $1,25 \mathrm{~A} \mathrm{a}$ & $0,50 \mathrm{~A} \mathrm{a}$ & $4,50 \mathrm{~A} \mathrm{a}$ \\
\hline \multirow[t]{2}{*}{ Chlorpyrifos (EC) } & $0,00 \mathrm{~A}$ a & $4,00 \mathrm{~A}$ a & $18,25 \mathrm{~B} \mathrm{~b}$ \\
\hline & \multicolumn{3}{|c|}{ Fitotoxicidade aos 28 DAA } \\
\hline Sem inseticida & $0,00 \mathrm{~A} \mathrm{a}$ & $0,00 \mathrm{~A} \mathrm{a}$ & $0,50 \mathrm{~A} \mathrm{a}$ \\
\hline Chlorpyrifos (CE) & $0,00 \mathrm{~A} \mathrm{a}$ & $0,50 \mathrm{~A} \mathrm{a}$ & $13,50 \mathrm{~B} \mathrm{~b}$ \\
\hline Lambdacyhalothrin & $0,00 \mathrm{~A}$ a & $0,00 \mathrm{~A}$ a & $0,00 \mathrm{~A}$ a \\
\hline Thiamethoxan + lambdacyhalothrin & $0,00 \mathrm{~A} \mathrm{a}$ & $0,00 \mathrm{~A} \mathrm{a}$ & $2,00 \mathrm{~A} \mathrm{a}$ \\
\hline Chlorpyrifos (EC) & $0,00 \mathrm{~A} \mathrm{a}$ & $1,25 \mathrm{~A} \mathrm{a}$ & $11,50 \mathrm{~B} \mathrm{~b}$ \\
\hline
\end{tabular}

Médias seguidas por letras maiúsculas iguais na coluna (comparação dos inseticidas para cada herbicida), ou minúsculas iguais na linha (comparação dos herbicidas para cada inseticida), não diferem entre si pelo teste de Tukey a 5\%. CE: concentrado emulsionável; EC: emulsão concentrada. 
Tabela 3. Médias da altura de plantas no florescimento (m), massa de 1.000 grãos (g) e rendimento de grãos (t ha $\left.{ }^{-1}\right)$ do milho híbrido (AGN 2012), corrigido para 14\% de umidade, submetido à aplicação de diferentes misturas de herbicidas e inseticidas. Piracicaba, 2004

\begin{tabular}{|c|c|c|c|c|c|c|c|}
\hline \multirow{2}{*}{ Herbicida } & \multirow{2}{*}{ Inseticida } & \multicolumn{2}{|c|}{ Altura } & \multicolumn{2}{|c|}{ Massa de 1.000 grãos } & \multicolumn{2}{|c|}{ Rendimento } \\
\hline & & $\mathrm{T}^{1}$ & $\mathrm{NT}^{1}$ & $\mathrm{~T}$ & NT & $\mathrm{T}$ & NT \\
\hline \multirow{4}{*}{ Sem hebricida } & Chlorpyrifos (CE) & $1,53 \mathrm{a}$ & $1,59 \mathrm{a}$ & 337,86 a & 331,19 a & $9,25 \mathrm{a}$ & $9,18 \mathrm{a}$ \\
\hline & Lambdacyhalothrin & $1,57 \mathrm{a}$ & $1,52 \mathrm{a}$ & $336,77 \mathrm{a}$ & 346,48 a & $9,82 \mathrm{a}$ & $8,89 \mathrm{a}$ \\
\hline & Thiamethoxan + lambda. & $1,59 \mathrm{a}$ & $1,60 \mathrm{a}$ & 347,90 a & $349,30 \mathrm{a}$ & $10,02 \mathrm{a}$ & 9,61 a \\
\hline & Chlorpyrifos (EC) & $1,50 \mathrm{a}$ & $1,51 \mathrm{a}$ & 340,15 a & 340,62 a & $10,23 \mathrm{a}$ & 9,11 a \\
\hline \multirow{4}{*}{ Mesotrione + atrazine } & Chlorpyrifos (CE) & $1,52 \mathrm{a}$ & $1,57 \mathrm{a}$ & 332,16 a & 333,41 a & 9,13 a & 9,33 a \\
\hline & Lambdacyhalothrin & $1,52 \mathrm{a}$ & $1,47 \mathrm{a}$ & $351,94 \mathrm{a}$ & 343,21 a & $9,69 \mathrm{a}$ & $9,17 \mathrm{a}$ \\
\hline & Thiamethoxan + lambda. & $1,52 \mathrm{a}$ & $1,57 \mathrm{a}$ & $338,00 \mathrm{a}$ & 326,45 a & $10,20 \mathrm{a}$ & $9,96 \mathrm{a}$ \\
\hline & Chlorpyrifos (EC) & $1,55 \mathrm{a}$ & $1,50 \mathrm{a}$ & 340,11 a & 333,65 a & $8,52 \mathrm{a}$ & 8,05 a \\
\hline \multirow{4}{*}{$\begin{array}{l}\text { Nicolsulfuron } \\
+ \text { atrazine }\end{array}$} & Chlorpyrifos (CE) & $1,51 \mathrm{a}$ & $1,55 \mathrm{a}$ & 328,24 a & 341,40 a & 7,86 a & $9,10 \mathrm{a}$ \\
\hline & Lambdacyhalothrin & $1,50 \mathrm{a}$ & $1,50 \mathrm{a}$ & 346,11 a & 347,23 a & $9,34 \mathrm{a}$ & 9,13 a \\
\hline & Thiamethoxan + lambda. & $1,50 \mathrm{a}$ & $1,55 \mathrm{a}$ & 341,35 a & 344,76 a & 9,37 a & 10,09 a \\
\hline & Chlorpyrifos (EC) & $1,52 \mathrm{a}$ & 1,55 a & 344,61 a & 353,02 a & 8,79 a & $10,41 \mathrm{a}$ \\
\hline \multicolumn{2}{|l|}{$F_{(\text {Herb } x \text { Ins } x \text { Test })}$} & \multicolumn{2}{|c|}{$0,47^{\mathrm{ns}}$} & \multicolumn{2}{|c|}{$0,63^{\mathrm{ns}}$} & \multicolumn{2}{|c|}{$0,74^{\mathrm{ns}}$} \\
\hline \multicolumn{2}{|l|}{ C.V. (\%) Parcelas } & \multicolumn{2}{|c|}{5,54} & \multicolumn{2}{|c|}{4,14} & \multicolumn{2}{|c|}{13,17} \\
\hline C.V. (\%) Subparcelas & & \multicolumn{2}{|c|}{3,38} & \multicolumn{2}{|c|}{4,85} & \multicolumn{2}{|c|}{14,85} \\
\hline \multicolumn{2}{|l|}{ DMS } & \multicolumn{2}{|c|}{0,07} & \multicolumn{2}{|c|}{23,28} & \multicolumn{2}{|c|}{1,96} \\
\hline
\end{tabular}

( $\left.{ }^{1}\right)$ Comparação das médias T x NT, na linha, para cada parâmetro, realizada por meio do teste de Tukey a $5 \%$; ${ }^{\text {ns }}$ - interação nãosignificativa. T: parcelas tratadas com os defensivos; NT: parcelas não-tratadas (testemunha dupla); lambda.: lambdacyhalothrin; CE: concentrado emulsionável; EC: emulsão concentrada.

Em algumas pesquisas nota-se que chuvas durante ou anteriores à aplicação do herbicida aumentam a injúria, pois favorecem a maior absorção do inseticida e do herbicida (MORTON et al. 1991; Kapusta e KRAusz, 1992). Contudo, condições climáticas favoráveis para o desenvolvimento da cultura após a aplicação do herbicida, favorecem sua recuperação (Peixoto e Ramos, 2002). Durante o ano agrícola do experimento, ocorreram precipitações pluviais e temperaturas adequadas para o desenvolvimento normal da cultura. Os parâmetros climáticos podem ter desfavorecido a interação dos inseticidas com os herbicidas, pois possibilitaram seu rápido metabolismo (Figura 1).

Outros fatores também podem afetar a interação entre duas classes de defensivos quando aplicados em misturas, dentre eles o processo de absorção, translocação e metabolismo dentro da planta (HARtzler et al., 2002); no entanto, os mecanismos de seletividade também são fundamentais. Segundo VAN EERD et al. (2003), alguns agroquímicos podem estimular ou prejudicar a ação do complexo enzimático P-450, que é responsável por uma das fases de metabolismo de herbicidas em plantas, fato que pode afetar seu crescimento e desenvolvimento.
Uma explicação para a interação de sulfoniluréias e organo-fosforados é que os metabólitos do terbufós na planta de milho inibem o citocromo P-450, responsável pela metabolização do nicosulfuron; sendo assim, acumula-se provocando injúrias (BAERG e Barrett, 1993; SiMINSZKY et al., 1995; Hinz et al., 1997). Acredita-se que as diferenças de intensidade da interação do terbufós e do chlorpyrifos com o nicosulfuron possam estar relacionadas com diferenças quanto à intensidade de inibição da enzima P-450 ou com a sistemicidade desses produtos.

O mecanismo de seletividade do mesotrione ainda não está completamente esclarecido, contudo há evidências significativas de que seja resultado de metabolismo diferencial entre a cultura do milho e as plantas daninhas (Mitchell et al., 2001). Os sintomas de fitotoxicidade decorrentes da interação do mesotrione com chlorpyrifos sugerem que o herbicida é metabolizado por um mecanismo semelhante ao que detoxifica o nicosulfuron, contudo, por características diferenciais de cada molécula, os danos foram menos severos. A atrazine, por sua vez, possui outra rota de detoxificação dentro das plantas. A seletividade dessa molécula às plantas de milho é, principalmente, conseqüência da conjugação do herbicida com a 
glutationa (GHS) (JABlonKAI e Hatzios, 1993), porém outros processos metabólicos também podem contribuir consideravelmente para a seletividade (Esser et al., 1975). Assim, não tem sido relatados casos de interação entre a atrazine e os inseticidas organo-fosforados.

\section{CONCLUSÃO}

1. A aplicação em pós-emergência da mistura dos herbicidas nicosulfuron + atrazine e mesotrione + atrazine com inseticidas da classe dos organofosforados causou intoxicação em plantas de milho (cloroses - amarelecimento ou branqueamento), principalmente para o herbicida nicosulfuron.

2. Não foram observadas diferenças significativas entre os tratamentos herbicidas ou inseticidas e as testemunhas com relação à altura de plantas, massa de mil grãos e rendimento.

\section{AGRADECIMENTOS}

Os autores agradecem à Fundação de Amparo à Pesquisa do Estado de São Paulo (FAPESP) pela bolsa de mestrado concedida ao primeiro autor.

\section{REFERÊNCIAS}

BAERG, R.J.; BARRET, M. Insecticide modifications of cytochrome P450 mediated herbicide metabolism. Weed Science, Champaign, v. 48, p.70, 1993.

BIEDIGER, D.L.; BAUMANN, P.A.; WEAVER, D.N.; CHANDLER, J.M. MERKLE, M.G. Interactions between primisulfuron and selected soil applied insecticides in corn (Zea mays). Weed Technology, Champaign, v.6, p.807-812, 1992.

COLE, D.J. Detoxification and activation of agrochemicals in plants. Pesticide Science, Southampton, v.42, p.209-222, 1994.

CONSTANTIN, J.; OLIVEIRA JÚNIOR, R.S.; MARCHIORI JÚNIOR, O.; CARREIRA, S.A.M.; FAGLIARI, J.R.; PAGLIARI, P.H.; PIVETTA, J.P. Seletividade de isoxaflutole aplicado isoladamente e conjuntamente com diuron+hexazinone em cana-deaçúcar em pré e pós emergência. STAB - Açúcar, Álcool e Subprodutos, Piracicaba, v.22, n.1, p.34-38, 2003.

CRUZ, I. Manejo de pragas na cultura do milho. In: FANCELLI, A.L; DOURADO-NETO, D. (Eds.). Milho: estratégias de manejo para alta produtividade. Piracicaba/SP: ESALQ/USP/LPV, 2003. p.19-46.

EMBRAPA. Sistema brasileiro de classificação de solos. Brasília, 1999. 412p.
ESSER, H.O.; DUPUIS, G.; EBERT, E.; VOGEL, C.; MARCO, G.J. s-Triazines. In: KEARNEY, P.C.; KAUFMAN, D.D. (Eds.). Herbicides: chemistry, degradation, and mode of action. 2ed. New York: Dekker, 1975. v.1., p.129-208.

FAGLIARI, J.R.; OLIVEIRA JÚNIOR, R.S.; CONSTANTIN, J. Métodos de avaliação da seletividade de herbicidas para a cultura da cana-de-açúcar. Acta Scientiarum, Maringá, v.23, n.5, p.1229-1234, 2001.

GASSEN, D.N. O risco da mistura de herbicidas com inseticidas em milho. In: GASSEN, D.N. (Ed.) Informativos técnicos Cooplantio. Passo Fundo: Aldeia Norte Editora, 2002. p.125-128.

HARTZLER, B.; PRINGNITZ, B.;OWEN, M. Interactions between ALS-herbicides and organophosphate insecticides. Integrated crop management, Ames, Iowa. 22 may 2000. Dissponível em: http:/ / www.ipm.iastate.edu/ipm/icm/2000/5-22-2000/ interaction.html. Acesso em 17 de dezembro de 2002.

HINZ, J.R.R.; OWEN, M.D.K.; BARRETT, M. Nicosulfuron, primisulfuron, and bentazon hydroxylation by corn (Zea mays), woolly cupgrass (Eriochloa villosa), and shattercane (Sorghum bicolor) cytochrome P-450. Weed Science, Lawrence, v.45, p.474480, 1997.

JABLONKAI, I.; HATZIOS, K.K. In vitro conjugation of chloroacetalinide herbicides and atrazine with thiols and contribution of nonenzymatic conjugation to their glutathionemediated metabolism in corn. Journal of Agricultural, Food and Chemistry, Davis, v.41, p.1736-1742, 1993.

KAPUSTA, G.; KRAUSZ, R.F. Interaction of terbufós and nicosulfuron on corn (Zea mays). Weed Technology, Champaign, v.6, p.999-1003, 1992.

KWON, C.S.; PENNER, D. The interaction of insecticides with herbicide activity. Weed Technology, Champaign, v.9, p.119124, 1995.

LÓPEZ-OVEJERO, R.F.; FANCELLI, A.L.; DOURADONETO, D.; GARCÍA y GARCÍA, A.; CHRISTOFFOLETI, P.J. Seletividade de herbicidas para a cultura de milho (Zea mays) aplicados em diferentes estádios fenológicos da cultura. Planta Daninha, Viçosa, v.21, n.3, p.413-419, 2003a.

LÓPEZ-OVEJERO, R.F.; CHRISTOFFOLETI, P.J.; NICOLAI, M; BARELA, J.F. Manejo de plantas daninhas na cultura do milho. In: FANCELLI, A.L; DOURADO-NETO, D. (Eds.). Milho: estratégias de manejo para alta produtividade. Piracicaba/SP: ESALQ/USP/LPV, 2003b. p.47-79.

MITCHELL, G.; BARTLETT, D.W.; FRASER, T.E.M.; HAWKES, T.R.; HOLT, D.C.; TOWSON, J.K.; WICHERT, R.A. Mesotrione: a new selective herbicide for use in maize. Pest Management Science, Hoboken, v.57, p.120-128, 2001.

MORTON, C.A.; HARVEY, R.G.; KELLS, J.J.; LEUSCHEN, W.E.; FRITZ, V.A. Effect of DPX-V9360 and terbufos on field and sweet corn (Zea mays) under three environments. Weed Technology, Champaign, v.5, p.130-136, 1991. 
MORTON, C.A.; HARVEY, R.G.; WEDBERG, J.L.; KELLS, J.J.; LANDIS, D.A.; LEUSCHEN, W.E. Influence of corn rootworm insecticides on the response of field corn (Zea mays) to nicosulfuron. Weed Technology, Champaign, v.5, p.130-136, 1994.

OLIVEIRA JÚNIOR, R.S. Seletividade de herbicidas para culturas e plantas daninhas. In. OLIVEIRA JÚNIOR, R.S.; CONSTANTIN, J. (Coords.). Plantas daninhas e seu manejo. Guaíba: Agropecuária, 2001. p.291-314.

OMOTO, C.; RISCO, M.D.M.; SCHMIDT, J.B.; ZUCCHI, T.D. Resistência de pragas a inseticidas no MIP na cultura do milho. In: CONGRESSO NACIONAL DE MILHO E SORGO. 24., 2002, Florianópolis. Anais... Florianópolis: Embrapa Milho e Sorgo; Epagri, 2002. 1 CD-ROM.

PEIXOTO, C. M.; RAMOS, A.A. Milho: manejo de herbicida. Pelotas: Cultivar, 2002. 10p. (Cultivar Grandes Culturas, 42)

RAIJ, B.V.; CANTARELLA, H. Milho para grãos e silagens. In: RAIJ, B.V.; CANTARELLA, H.; QUAGGIO J.A.; FURLANI A.M.C. (Eds.). Recomendações de adubação e calagem para o Estado de São Paulo. Instituto Agronômico: Campinas, 1996. p 56-59. (Boletim Técnico 100)
SILVA, A.A.; FREITAS, F.M.; FERREIRA, L.R.; JAKELAITIS, A. Efeitos de mistura de herbicidas com inseticidas sobre a cultura do milho, as plantas daninhas e a lagarta-do-cartucho. Planta Daninha, Viçosa, v.23, n.3, p.517-525, 2005a.

SILVA, A.A.; FREITAS, F.M.; FERREIRA, L.R.; JAKELAITIS, A.; SILVA, A.F. Aplicações seqüenciais e épocas de aplicação de herbicidas em mistura com chlorpirifós no milho e em plantas daninhas. Planta Daninha, Viçosa, v.23, n.3, p.527-534, 2005b.

SIMINSZKY, B.; CORBIN, F.T.; SHELDON, Y. Nicosulfuron resistance and metabolism in terbufos- and naphthalic anhydride-treated corn. Weed Science, Champaigne, v.43, p.163-168, 1995.

VAN EERD, L.L.; HOAGLAND, R.E.; ZABLOTOWICZ, R.M.; HALL, J.C. Pesticide metabolism in plants and microorganisms. Weed Science, Lawrence, v.51, p.472-495, 2003. 